\title{
Hemothorax Revealing Aortic Aneurism and Aortic Dissection
}

\author{
Fatma Chermiti Ben Abdallah", Houda Gharsalli, Hamida Kwas, \\ Sofia Taktak, Amel Chtourou, Ali Ben Kheder \\ Pneumology IV department, Abderrahman Mami Hospital, Ariana, Tunisie \\ Email: ${ }^{*}$ cherabdallah@yahoo.fr
}

Received April 2, 2013; revised May 5, 2013; accepted May 15, 2013

Copyright (C) 2013 Fatma Chermiti Ben Abdallah et al. This is an open access article distributed under the Creative Commons Attribution License, which permits unrestricted use, distribution, and reproduction in any medium, provided the original work is properly cited.

\begin{abstract}
Hemothorax caused by rupture of aortic aneurysm or aortic dissection is an uncommon manifestation and carries a high mortality rate. We report two cases of 75- and 80-year-old men, who were referred to our department for exploration of a left pleural opacity. Thoracentesis produced a hemorrhagic fluid. The computed tomography scan revealed an aortic dissection type B of Stanford in the first case and an aneurysm of the descending thoracic aorta in the second patient. Emergency operation was performed in the first case but the patient died of multiple organ failure 48 hours after surgery. Aortic dissection or aneurysm can result in a hemothorax especially in patient with favourable conditions such as elderly patient and systemic hypertension. Diagnosis relies on computed tomography (CT) scan and echocardiography.
\end{abstract}

Keywords: Aortic Aneurysm; Aortic Dissection; Hemothorax; Computed Tomography Scan; Echocardiography

\section{Introduction}

Hemothorax complicating rupture of a large chest vessel is rare. It is essentially caused by rupture of an aneurysm or dissection of the aorta into the pleural cavity. It is a rare complication and its diagnosis is often delayed which results a high mortality.

\subsection{Case 1}

A 75-year-old man, smoking 60-pack-yrs, with a history of hypertension was admitted in our department for 3day duration of left chest pain associated with dyspnea and an episode of syncope. On examination, respiration rate was 28 breaths per minute; pulse rate, 100 beats per minute; and blood pressure, 110/60 $\mathrm{mmHg}$. Breath sounds were decreased at the left lung with dullness at percussion. A systolic murmur in the mitral area was noticed on heart examination. Auscultation of great vessels was normal. Peripheral pulses were palpated. The chest radiograph revealed a massive left-sided pleural effusion (Figure 1). The electrocardiogram showed normal sinus rhythm and lateral ventricular repolarization disorders. Laboratory tests revealed microcytic hypochromic anemia with haemoglobin level of $7 \mathrm{~g} / \mathrm{dl}$ and renal failure

${ }^{*}$ Corresponding author. (serum urea level: $20.85 \mathrm{mmol} / \mathrm{l}$, creatinine value: 200 umol/l). Clotting parameters were normal. Thoracentesis of the left pleural cavity yielded a bloody fluid. The chest computed tomographic (ct) scan confirmed the presence of a left-sided pleural effusion and showed a hematoma of the aortic arch and the descending aorta, periaortic hematoma and a pseudoaneurysm of the descending aorta (Figure 2). Trans-oesophageal echocardiography objectified a descending aortic dissection type B of stanford associated with a thombus of the false lumen. Urgent surgery was indicated and the patient had a left posterolateral thoracotomy with removal of clothed blood and pleural effusion drainage. Vascular replacement was not recommended because of advanced age and renal failure. A multi-system organ failure followed by the death of the patient occurred 48 hours after surgery.

\subsection{Case 2}

An 80-non smoker-year old-man, without medical history was hospitalized in our department for one month history of left-sided chest pain. On physical examination, signs of left pleural effusion were found. Blood pressure of the patient was $100 / 60 \mathrm{mmHg}$ and cardiac frequency 110 beats/minute. Auscultation of great vessels was normal. Chest radiograph revealed a left pleural effusion 


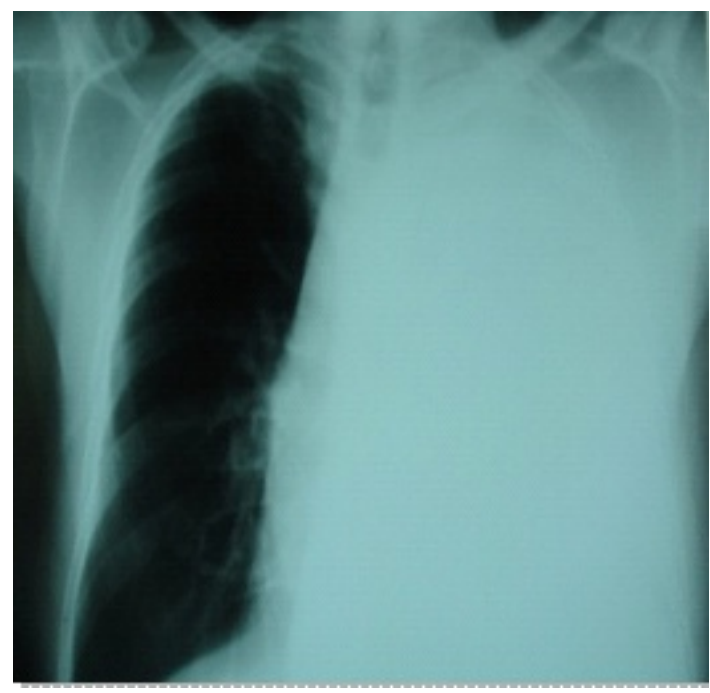

Figure 1. Chest radiograph showing left pleural opacity.

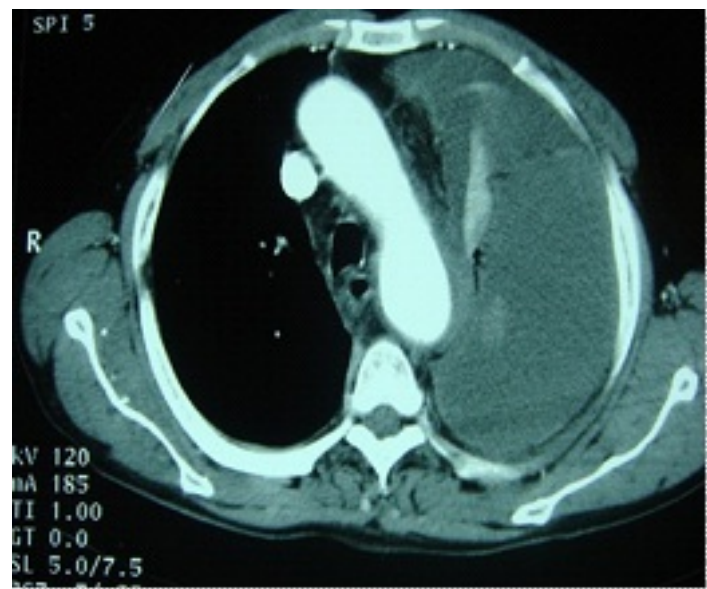

Figure 2. CT scan revealing aortic dissection with left-sided pleural effusion and hematoma of the aortic arch.

with deviation of the mediastinum to the controlateral side. The electrocardiogram showed normal sinus rhythm with diffuse repolarization disorders corresponding to epicardial ischemia. Clotting parameters and cardiac enzymes were normal. Thoracentesis revealed hemothorax. The chest CT scan performed urgently, objectified false aneurysm of the arch and descending thoracic aorta fistulized in the left pleural cavity (Figures 3(a) and (b)). Because of advanced aged and good tolerance, surgery was not indicated. He died after 8 months.

\section{Discussion}

Hemothorax is defined as pleural effusion with a haematocrit $\geq 50 \%$ that of blood [1]. The majority of haemothraces is due to penetrating trauma or is iatrogenic complicating invasive technique such as central venous catheters, pleural biopsy or thoracentesis. Others causes have been reported such as hemostasis disorders occur-

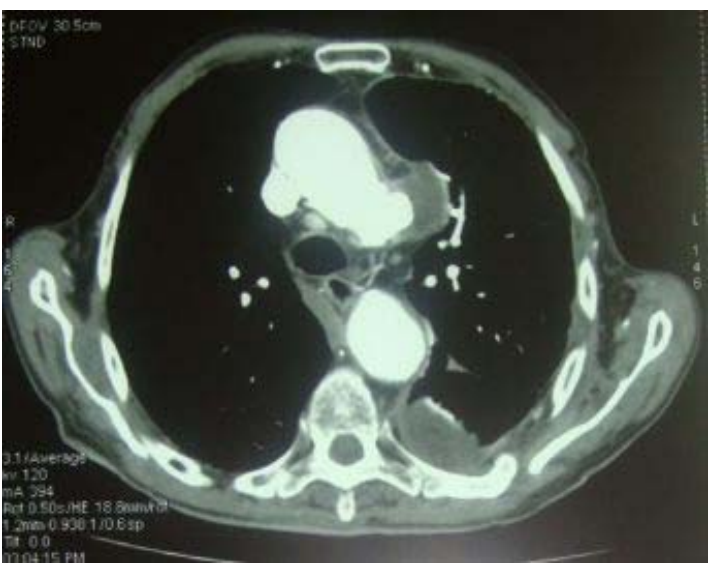

(a)

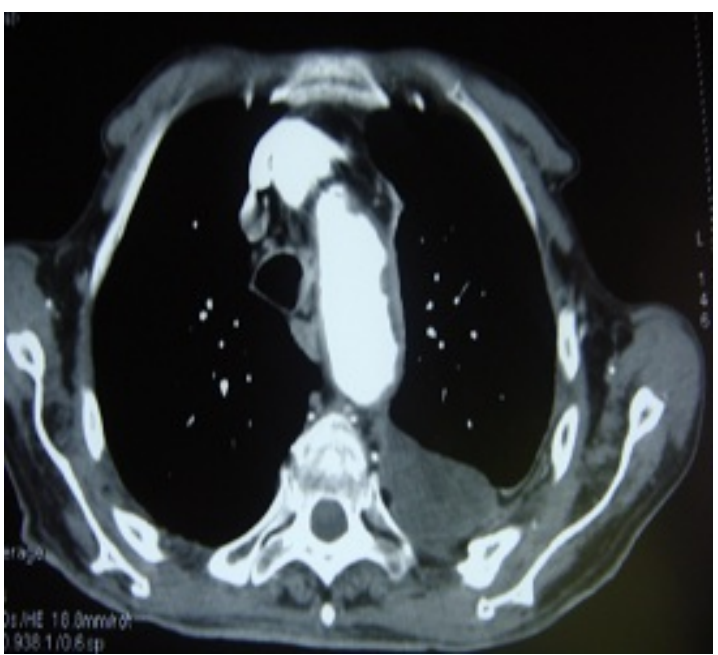

(b)

Figure 3. (a), (b) CT scan demonstrating false aneurysm of the arch and descending thoracic aorta fistulized in the left pleural cavity.

ring during anticoagulation therapy, hemophilia and thrombocytopenia [2]. Other rare etiologies have been also reported as pulmonary embolism with or without infarction, empyema and malignant pleural tumors [2]. The hemothorax secondary to rupture of a great chest vessel is uncommon. It is essentially aneurysm of the aorta, an aortic dissection or pulmonary arteriovenous malformations [2].

Hemothorax of vascular origin are often due to a rupture of the descending thoracic aorta, initially in the mediastinal and left pleural space due to the proximity of the pleural cavity [3], as was the case is in our patients. Rupture of the thoracic aorta in the right pleural cavity is rare $[4,5]$. The ascending thoracic aorta bleeds mainly into the pericardium [5]. Aortic dissection and aneurysm affect patients with risk factors such as hypertension, congenital aortic anomalies (aortic coarctation, bicuspid aortic valve disease.) and abnormal collagen tissue (Marfan syndrome, Ehlers-Danlos syndrome) [6]. A history of hypertension 
was reported by the first patient however no known risk factor has been reported by the second advanced aged patient.

Acute chest pain and hemodynamic signs are the classic presenting symptoms of aortic rupture. However, some elderly, as our second patient may remain asymptomatic, especially if the rupture occurs insidiously [4].

Radiological investigations are the key for diagnosis: chest $\mathrm{X}$ rays may show mediastinal enlargement, left pleural effusion, as we have observed in our patients and indistinct aortic contour [3,7]. Such radiologic anomalies should suggest the diagnosis if they occur in a patient at risk. The chest $\mathrm{CT}$ scan with contrasts the most common initial diagnostic test performed because it is commonly available in emergency departments and can be performed readily. It is the tool of choice for the diagnosis and management of patients with suspected aortic dissection [8]. MRI stills a reference technique. However, it is not appropriated in context of emergency [3].

Trans-oesophageal echocardiography is an investigation of first-line, feasible at the bedside even in an emergency situation [3].

Treatment depends on the type of lesions. For aneurysms, surgical treatment is indicated when patients become symptomatic. In the absence of surgery, radioclinical strict monitoring is necessary. Dissections of the ascending aorta (Stanford type A) must be operated in an emergency, while surgery of descending aortic dissections (Stanford type B) are made in cases of acute complications [9]. Thoracic drainage in these situations is not recommended because it can lead hemodynamic instability [5].

The course is variable, ranging between the disappea ring of pleural effusion to death [2]. In our first patient, there were clinical and biological signs (anemia and renal failure) of poor prognosis. The second patient, for whom surgery was not indicated, died after 8 months.

\section{Conclusion}

Rupture of aortic dissection and aortic aneurysm in the left pleural cavity is one of the various etiologies of hemothorax, which should be considered in patients with risk factors. The absence of thoracic pain does not exclude this diagnosis based essentially on imaging, particularly CT scan and trans-oesophageal echocardiogra- phy. The treatment and the course depend mainly on the severity of the initial clinical presentation, the type and the extent of aortic lesion and the patient history.

\section{REFERENCES}

[1] S. Little, J. Johnson, B. Y. Moon and S. Mehta, "Painless Left Hemorrhagic Pleural Effusion," Chest, Vol. 116, No. 5, 1999, pp. 1478-1480. doi:10.1378/chest.116.5.1478

[2] T. Langin, P. E. Kelkel and D. Villemot, "Left Hemothorax, a Complication of a Dissecting Aneurysm of the Descending Thoracic Aorta," Revue Des Maladies Respiratoires, Vol. 11, No. 1, 1994, pp. 74-76.

[3] S. Bousnina, I. Zendah, K. Marniche, H. Racil, S. Kachboura, M. L. Megdiche and A. Chabbou, "Left Hemothorax Caused by Aortic Dissection," Revue de Pneumologie Clinique, Vol. 63, No. 2, 2007, pp. 119-122. doi:10.1016/S0761-8417(07)90113-3

[4] J. Josserand, B. Boura, L. Yakhou, P. Albaladejo and J. Marty, "Rupture of a Thoracic Aortic Aneurysm into the Right Pleural Cavity," Annales Françaises d'Anesthésie et de Réanimation, Vol. 25, No. 9, 2006, pp. 997-999. doi:10.1016/j.annfar.2006.06.015

[5] F. Baharloo, R. Verhelst, P. Collard and T. Pieters, "Rupture of Aortic Aneurysm with Right-Sided Haemothorax," European Respiratory Journal, Vol. 13, No. 2, 1999, pp. 465-467. doi:10.1183/09031936.99.13246599

[6] E. M. Larson and W. D. Edwards, "Risk Factors for Aortic Dissection: A Necropsy Study of 161 Cases," The American Journal of Cardiology, Vol. 53, No. 6, 1984, pp. 849-855. doi:10.1016/0002-9149(84)90418-1

[7] S. F. Miller, H. R. Shah, R. S. Pritchard and E. C. Buckner, "CT Diagnosis of Right Hemothorax Secondary to Ruptured Thoracoabdominal Aneurysm: Case Report," CardioVascular and Interventional Radiology, Vol. 12, No. 6, 1990, pp. 326-328. doi:10.1007/BF02575431

[8] F. Özkan, E. Akpına, T. Serter, A. Özyüksel and T. Hazırolan, "Ruptured Type B Aortic Dissection Presenting with Right Hemothorax," Diagnostic and Interventional Radiology, Vol. 14, No. 1, 2008, pp. 6-8.

[9] W. Slisatkorn, P. Laksanabunsong and P. Thongcharoen, "Ruptured Right Aortic Arch Aneurysm," Asian Cardiovascular and Thoracic Annals, Vol. 12, No. 4, 2004, pp. 360-362. doi:10.1177/021849230401200417 